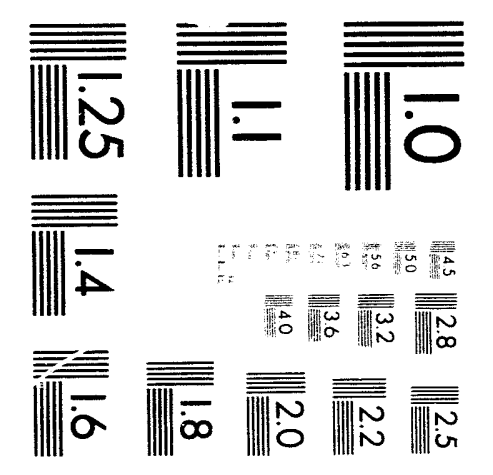



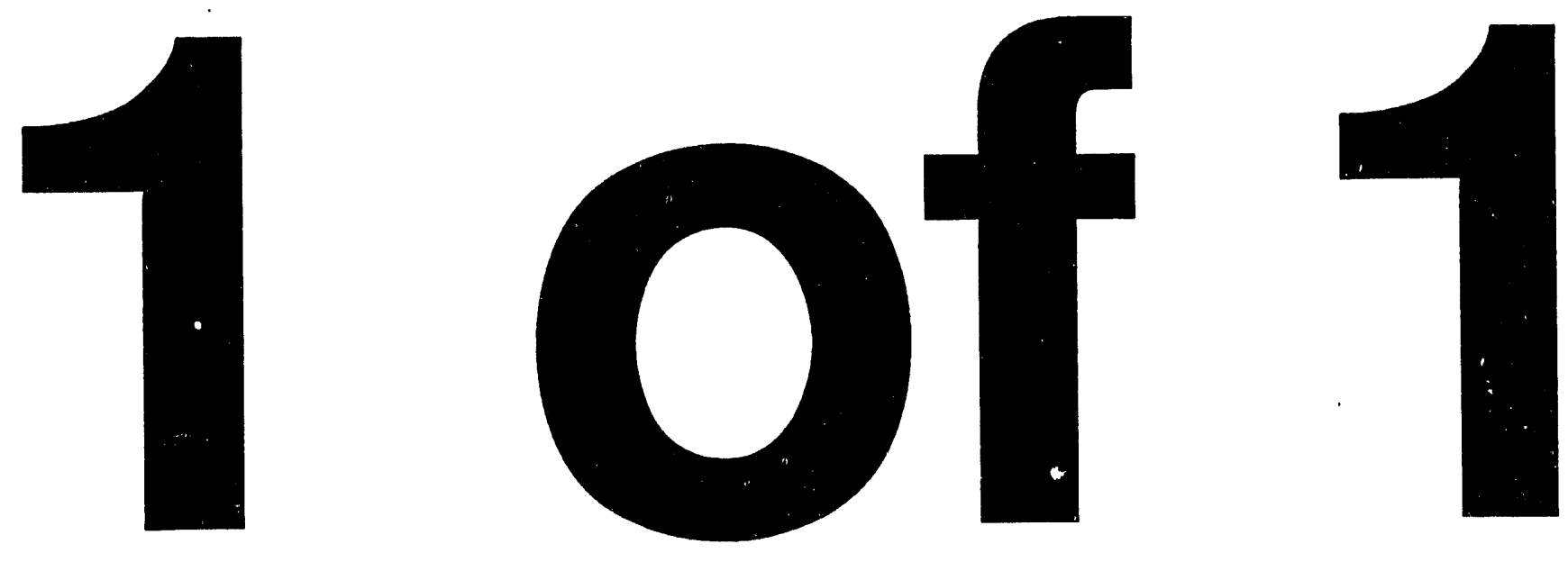


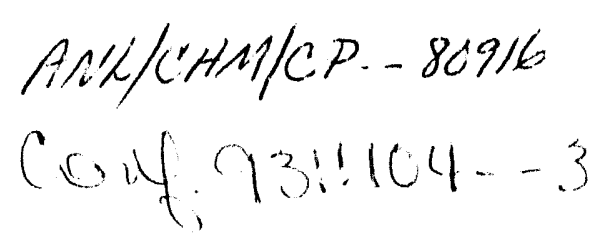

Sputtering of Neutral and Ionic Indium Clusters*

OCT 191993

\author{
Z. Ma, S.R. Coon, ${ }^{1}$ W.F. Calaway, M.J. Pellin, D.M. Gruen \\ and E.I. von Nagy-Felsobuki ${ }^{2}$
}

os ri

Materials Science, Chemical Technology, and Chemistry Divisions, Argonne National Laboratory

Argonne, IL 60439

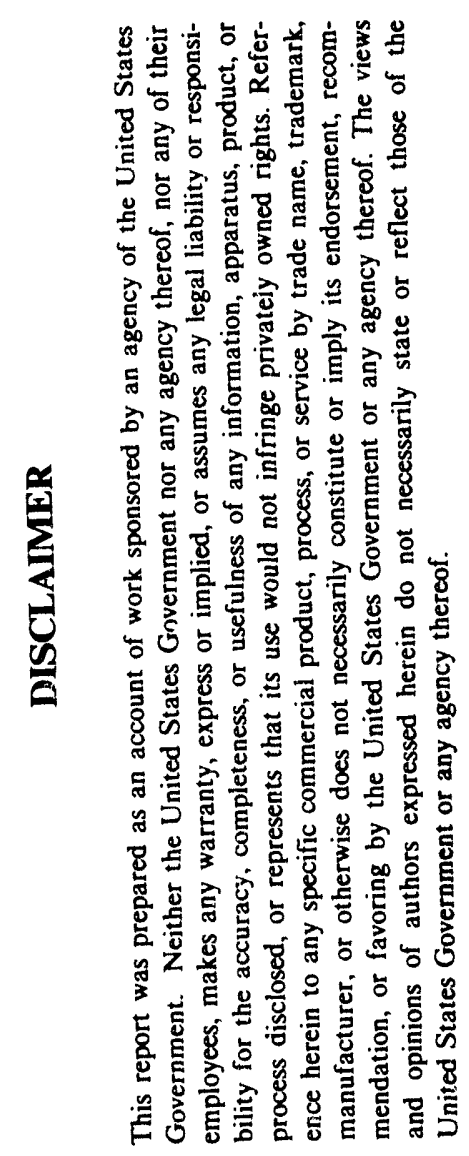

\author{
Submitted to the \\ Journal of Vacuum Science and Technology A \\ for inclusion in the Proceeding of the \\ 40th Annual American Vacuum Society Symposium \\ Orlando, Florida \\ November 15-19, 1993
}

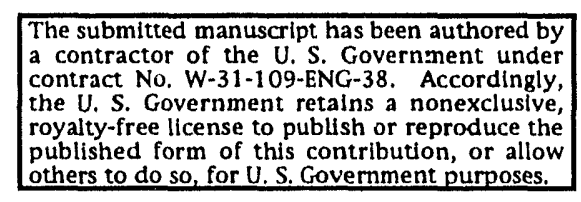

1 Present address: Department of Chemistry, The University of Iowa, Iowa City, Iowa 52242

2 Permanent address: Department of Chemistry, The University of Newcastle, Callaghan 2283 NSW, Australia.

* Work supported by the US Department of Energy, BES-Materials Sciences, under Contract W-31-109-ENG-38. 


\title{
Sputtering of Neutral and Ionic Indium Clusters*
}

\author{
Z. Ma, S.R. Coon, ${ }^{1}$ W.F. Calaway, M.J. Pellin, D.M. Gruen \\ and E.I. von Nagy-Felsobuki ${ }^{2}$ \\ Materials Science, Chemical Technology, and Chemistry Divisions, \\ Argonne National Laboratory \\ Argonne, IL 60439
}

\begin{abstract}
Secondary neutral and secondary ion cluster yields were measured during the sputtering of a polycrystalline indium surface by normally incident $\sim 4 \mathrm{keV} \mathrm{Ar}^{+}$ions. In the secondary neutral mass spectra, indium clusters as large as $\operatorname{In}_{32}$ were observed. In the secondary ion mass spectra, indium clusters up to $\operatorname{In}_{18}^{+}$were recorded. Cluster yields obtained from both the neutral and ion channel exhibited a power law dependence on the number of constituent atoms, $\mathrm{n}$, in the cluster, with the exponents measured to be -5.6 and -4.1 , respectively. An abundance drop was observed at $n=8,15$, and 16 in both the neutral and ion yield distributions suggesting that the stability of the ion (either secondary ion or photoion) plays a significant role in the observed distributions. In addition, our experiments suggest that unimolecular decomposition of the neutral cluster may also plays an important role in the measured yield distributions.
\end{abstract}

1 Present address: Department of Chemistry, The University of Iowa, Iowa City, Iowa 52242

2 Permanent address: Department of Chemistry, The University of Newcastle, Callaghan 2283 NSW, Australia.

* Work supported by the US Department of Energy, BES-Materials Sciences, under Contract W-31-109-ENG-38. 


\section{Introduction}

In order to understand the sputtering process and to develop trace analysis techniques that are capable of characterizing the surface composition of solids to parts per billion [1-2] additional studies are needed to compare the yields of neutral and ionic clusters emitted from surfaces under ion bombardment. In the case of oxygen adsorbed on metal surfaces [3] or for oxide materials [4], ions can be a substantial fraction of the sputtered flux. However, in the case of clean metals and semiconductors [5], it is well known that the neutral atoms are far more dominant. Only recently has it been possible to detect neutrals with high selectivity and sensitivity due to the development of postionization techniques using lasers.[1-2, 6-8] With sufficient laser power, ionization efficiencies near $100 \%$ are achievable, which is well above the $1 \%$ ionization efficiency found using post-ionization methods involving plasmas [9] or electron beams.[10]

Laser postionization secondary neutral mass spectrometry (SNMS) has recently been used in this laboratory to measure sputtering yields of neutral clusters as a function of cluster size from polycrystalline copper $[6,11]$ and aluminum $[11,12]$ surfaces. The measured yields were found to display a power law dependence on the constituent atom number.[11] A similar measurement on silver also shows a power law dependence.[13] Recently, we have shown that the slope for such power law plots can be correlated with sputtering yield.[11,14] Indium was chosen to continue these studies because the In sputtering yield is large, and because In as well as clusters of In are one photon ionizable with $193 \mathrm{~nm}$ photons [15] from an ArF excimer laser. Our previous experiments with $\mathrm{Cu}$ and $\mathrm{Al}$ suggest that single photon ionization reduces photofragmentation and thus simplifies spectral interpretation.[16]

So far, little is known about the neutral cluster yield distributions from an indium surface. Most of the recent work on indium has focused on the ion/neutral yield distribution of the atom and dimer. For example, using multiphoton resonance ionization (MPRI) and secondary ion mass spectrometry (SIMS), Kimock et al. have shown that the In and $\mathrm{In}_{2}$ yields decrease with oxidation, while the $\operatorname{In}^{+}$and $\operatorname{In}_{2}^{+}$yields increase dramatically (although the $\mathrm{In}_{2} / \mathrm{In}$ ratio remained similar to the $\mathrm{In}_{2}^{+} / \mathrm{In}^{+}$ratio).[17-18] Using a resonant two-color, two-photon 
ionization scheme, Craig et al. [19] studied the photofragmentation of sputtered indium dimer, giving evidence that the lowest excited state of the indium atom ${ }^{2} \mathrm{P}_{3 / 2}$, was not significantly populated by the sputtering process, but rather by laser-induced photodissociation of vibronically excited $\mathrm{In}_{2}$. No measurement for neutral indium clusters larger than dimer have been made.

Irion et al [20] recorded a number of SIMS spectra of polycrystalline surfaces (including indium) using a $20 \mathrm{keV} \mathrm{Xe}{ }^{+}$ion beam configured to a narrow-band Fourier transform mass spectrometer. Although ionic clusters containing up to 37 indium atoms $(\mathrm{m} / \mathrm{z}=$ 4249.45 amu) were measured with high mass resolution, the study reported no quantitative yield distribution data. King and Ross [21] measured the cluster yield distribution for In ions and the unimolecular dissociation rates for each ion up to $\operatorname{In}_{15}^{+}$. The large dissociation rates of In $_{8}^{+}$and $\operatorname{In}_{15}^{+}$and other anomalous intensities were attributed to the shell model.

For the sputtering of clean metal surfaces, the bond energies of the ejected clusters are typically less than the surface binding energy of a single atom. This is true for indium where the heat of sublimation is reported to be $2.52 \mathrm{eV} \mathrm{[22],} \mathrm{and} \mathrm{the} \mathrm{measured} \mathrm{binding} \mathrm{energy} \mathrm{for} \mathrm{the}$ indium dimer in its ground electronic state ${ }^{3} \Pi_{u}\left(\mathrm{O}_{\mathrm{u}}^{-}\right)$is $0.87 \mathrm{eV}$ [23]. Thus, it is surprising that substantial clusters are observed during sputtering. Quantal interactions are subtle and so mechanisms describing the ejection of these molecules still utilize an array of crude approximations. Models describing cluster emission from metals surface include multiple collision [24], recombination [25], correlated emission [26] and unimolecular decomposition of a vibrationally excited volume [27] formalisms. These models have been found wanting in predicting the experimental kinetic energy distributions of neutral clusters. For example, in the case of sputtered neutral clusters of aluminum [12,28], copper [6,11] and silver [29], experimental kinetic eneroy distribution curves exhibit high energy tails which are not as steep as predicted by the multiple collision model. In all cases, there are more high energy clusters detected experimentally than predicted by theory. Further, it has been demonstrated [30] that sputtering of an oxidized surface (rather than a clean surface) results in the ejection of ions and 
clusters in excited vibronic states and so the sputtered flux is not populated according to a Boltzmann distribution.

\section{Experimental}

Experiments were performed using the SARISA IV instrument developed in this laboratory [1-2]. This apparatus can operate as either a SNMS or SIMS machine. While it is possible to collect SNMS and SIMS data quasi simultaneously with this instrument via interleaving, the two types of data reported here were collected in separate experiments. For sputtering, a Colutron ion gun was employed that produced approximately $3 \mu \mathrm{A}$ of current. The ion beam was pulsed and focused in a $300 \mu \mathrm{m}$ spot onto a polycrystalline Marz grade indium foil. In all the experiments, a normal incidence $5 \mathrm{keV}$ (reference to ground) $\mathrm{Ar}^{+}$ion beam was used. All experiments were performed under ultra high vacuum conditions (base pressure of $4 \times 10^{-8} \mathrm{~Pa}$ ). To further insure surface cleanliness, the indium target was rastered with an ion beam in a $2 \mathrm{~mm}$ by $2 \mathrm{~mm}$ pattern for at least 30 minutes at the beginning of each day and again for at least one minute prior to every data acquisition.

Secondary ions or photoionized secondary neutrals were extracted and analyzed using the energy- and angle-refocussing time-of-flight (EARTOF) mass spectrometer that is a part of SARISA IV. The time dispersed (mass separated) ions were detected by a dual microchannel plate assembly in a chevron arrangement. The signal arising from the detector is either digitized directly (charge integrated) or pulse counted, depending on the intensity of the signal. A number of calibration spectra were taken under the same experimental conditions to obtain reliable conversion factors between the two modes of data collection and also to quantify the different detector gains which were used in various measurements. An important design feature of the EARTOF, when determining relative signal intensities over a large dynamic range, is the ability to electrostatically deflect a portion of the mass spectrum away from the detector. This allows high channel plate gains to be employed for the measurement of weak signals without overloading the detector with signals from intense species. 
For neutral species detection, the data collection sequence was initiated with a $1 \mathrm{~ms}$ long ion pulse. To suppress noise arising from secondary ions, the target was pulsed to $1400 \mathrm{~V}$ for the duration of the ion pulse. This imparts an energy to the secondary ions which is too high for the band pass of the EARTOF energy analyzers (nominally, $1.0 \pm 0.1 \mathrm{keV}$ ). At the end of the ion pulse, the target potential is lowered to $1100 \mathrm{~V}$ so that photoions produced in the laser volume have the appropriate energy to reach the detector. At some predetermined time delay after the end of the ion pulse (usually greater than $500 \mathrm{~ns}$ ), the laser is fired into the region above the target surface photoionizing the neutral species that had previously been sputtered from the surface. Background spectra with the laser beam blocked showed no evidence of secondary ions. Spectra taken with the ion beam blocked indicated that there was no ablation of the sample by stray laser light.

The photoionization of the sputtered species was accomplished using a ArF excimer laser $(6.4 \mathrm{eV}, 193 \mathrm{~nm})$. The laser intensity was measured to be $4 \mathrm{~mJ} / \mathrm{pulse}$ at the chamber when run at $50 \mathrm{~Hz}$. A $500 \mathrm{~mm}$ focal length spherical lens was used to focus the beam. By adjusting the distance of the lens from the sputtered flux, an laser cross section of $1 \mathrm{~mm}$ wide by $2 \mathrm{~mm}$ high near the target was achieved. To confirm this and to assess the role of photofragmentation, power studies of the measured signal as a function of the laser beam intensity were undertaken for clusters containing up to 24 atoms. This is accomplished by recording mass spectra at various laser intensities using partially absorbing quartz plates to attenuating the laser bearn. A total of four plates were used, each reducing the laser power by a factor of 2, yielding a dynamic range in laser power between $\sim 0.5-10 \mathrm{MW} / \mathrm{cm}^{2}$.

For the SIMS measurements, spectra were recorded without firing the photoionizing laser and triggering the target pulser. In addition, the target potential was adjusted to $1050 \mathrm{~V}$ to ensure that the secondary ions have an imparted energy within the band pass of the energy analyzers. Although changing target potential does affect the energy of the primary ions that strike the target (3.6 keV for SNMS to $3.95 \mathrm{keV}$ for SIMS), the small difference should not significantly alter the yields. Also for SIMS measurements, the ion pulse width was reduced to 200 ns. since unlike SNMS the ion pulse duration determines the mass resolution. 
In pulsed laser postionization experiments, the measured signal is proportional to the number density of those particles inside the laser volume. Thus, to estimate the relative yields, the various mass peaks were integrated and normalized to the atomic yield. Separate velocity distribution measurements of sputtered indium clusters have shown that cluster energy distributions are generally all quite similar and peak nearly at the same energy[31], which is consistent with previous experiments $[6,11,12]$. Therefore the average velocity of the species can be approximated by $\mathrm{m}^{-0.5}$, where $\mathrm{m}$ is the mass of the cluster. The signal intensity relative to the saturated atom is therefore divided by the square root of the number of atoms in the cluster to convert the data from number density to flux.

\section{Results and Discussion}

The purpose of this investigation is to measure the abundance distribution of indium neutral and ion clusters in order to gain understanding of sputtering mechanisms. Figure 1 shows a composite laser postionized SNMS spectrum of sputtered polycrystalline indium recorded in the analog mode for clusters swaller than Ing and in the counting mode for the remaining clusters. The spectrum in fig. 1 is an average of 2000 laser shots. The maximum cluster size observed is $\operatorname{In}_{32}$. However, it should be pointed out that even bigger clusters are possibly formed during the sputtering process, but are not recorded by the microchannel plate detector due to the small momentum of these larger clusters.

Figure 2 shows the composite SIMS spectrum for indium. The maximum indium cluster ion observed is $\operatorname{In}_{18}^{+}$. It is difficult to accurately determine the ratio of the neutral to ion yields in these experiments due to differences in the extraction efficiency for neutrals and ions into a mass spectrometer. However, ion optics calculations for the SARISA instrument as well as previous measurements [32] allow an order of magnitude estimates to be made. It is estimated that the ion yield in fig. 2 is about four orders of magnitude smaller than the atom yield obtained from fig. 1. This is consistent with the fact that atomic species ejected from clean metal surfaces are dominantly in a neutral state.[5] In the SIMS spectra, the presence of potassium was observed via the $\mathrm{K}_{2}^{+}$and $\mathrm{InK}^{+}$ions, the latter peak showing the largest intensity 
in the spectrum. An expansion of the spectrum clearly shows the expected isotopic abundance in the case of $\mathrm{K}_{2}^{+}$. It is believed that the potassium originates from the filament of the Colutron ion source and is not being effectively suppressed in the mass filter. Potassium produces a very large secondary ion signal due to its low ionization potential. Thus, it is believed that the amount of potassium is quite small and does not significantly alter the yield data.

Log-log plots of yield versus cluster size for neutrals and ions are shown in fig. 3 and fig. 4 , respectively. Notice that in fig. 4 , the $\operatorname{In}_{10}^{+}$yield is not shown since a noise spike associated with the instrument interferes with the signal in this mass region. The relative intensities of the neutral clusters $\left(\operatorname{In}_{n}, n=2-32\right)$ with respect to the atom signal ranged from $10^{-2}$ to $10^{-9}$. While there are some intensity fluctuations, both neutral and ionic yields show a overall power law dependence on cluster size, with the exponents being -5.6 and -4.1 , respectively. The power law dependence for sputtered neutral species has been reported for the first time only recently.[11,14] Exponents for the neutral power law dependencies of neutral $\mathrm{Cu}_{n}[11], \mathrm{Al}_{\mathrm{n}}[11]$, and $\mathrm{Ag}_{\mathrm{n}}[29]$ clusters have been determined to be -7.8, -9.3 and -6.5 , respectively. The slopes of the power law dependencies have been shown to correlate with the sputtering yield, with the gradient of the log-log plot becoming less negative as the total sputtering yield of the metal increases.[11,14] The slope for the indium neutral clusters also fits this trend, given that the sputtering yield for In is 9.[33]

It is interesting to note that the exponent for the ionic power law plot is less negative than the exponent for the neutral plot. There are two possible explanations to account for the different slopes. The first one is related to the sputtering process, i.e., what fraction of ejected clusters is in the ionic state. As the cluster size increases, the ionization potential decreases, therefore the ionization probability, and thus, the number of clusters appearing in charged state, may increase, making the icn curve less steep than the neutral curve. Experimental results on the sputtering of $\mathrm{Ag}$ have shown that atoms and small clusters $(n<6)$ are preferentially sputtered as neutrals, while the ionic state dominates for clusters larger than $\mathrm{Ag}_{10}$.[29] The second possibility is the fragmentation of the neutral clusters prior to ionization. This also would 
produce the steeper exponent observed in the log-log plot of neutral cluster yields if larger clusters tend to fragment more than small clusters.

Since the neutral and ionic cluster signal intensities depends on a variety of experimental conditions, it is important to address what factors, and to what extent, the experimental results are affected in order to assess how precisely the experimental result reflect the true yields. Conditions specially related to our experiment are discussed in the following:

1. Surface Cleanliness: Atom and cluster signals were found to be sensitive to surface cleanliness and so, as detailed in the experimental description above, sputtering was employed to keep the surface clean. One indication that the surface was clean is found in the fact that there was no evidence of indium oxide or oxygen products being present in the sputtered neutral and ion flux. Without ion beam rastering of the surface, a substa.tial decrease in signal, especially for the dimer and other cluster was noticeable. The atom signal was less sensitive to surface contamination than the clusters. The $\mathrm{In}_{2} / \mathrm{In}$ signal ratio has the largest drop followed by $\mathrm{In}_{3} / \mathrm{In}$ and $\mathrm{In}_{4} / \mathrm{In}$. The change in relative signals was roughly equal for clusters larger than In5. Hence, rastering the surface prior to data collection as describe in the experimental section was deemed necessary in order to accurately measure the yields. This observation is consistent with previous studies[17, 34].

2. Unimolecular Decomposition: Because of the large amount of internal energy that may deposited into sputtered clusters, unimolecular decomposition is a natural outcome of the sputtering process. These energy-rich clusters cool by evaporation of a single atom, a dimer, or other clusters. Using molecular-dynamics simulation based on a many-body embeddedatom potential, Wucher [35] has shown that metal clusters sputtered by $\mathrm{keV} \mathrm{Ar}+$ ions contain an average internal energy of roughly $1 \mathrm{eV}$ per constituent atom. These high internal energy nascent clusters decompose into stable and nietastable species on a time scale of picoseconds after leaving the surface. It is not possible to experimentally detect this decomposition process since it occurs so rapidly and very near the solid surface. The actual experimentally measured yields can be thought of as the sum of cold nascent clusters and the dissociation products of hot nascent clusters. Unimolecular fragmentation of metastable species formed during the 
sputtering process are know to occur on a longer time scale, but it is difficult to separate this process from decomposition due to photofragmentation (see below).

3. Ionization potential (IP) and photoionization cross section: In order to convert cluster signals to yields data, it is important to completely ionize all the species in the laser volume. This is best accomplished using one photon ionization since the cross sections for one photon processes are high and photofragmentation is minimized. The IP of atomic indium has been reported as $5.76 \mathrm{eV}$.[37] Data on the IP of In clusters above $n=4$ have also been measured [15]. These results show an overall decreasing function with constituent atom number, $\mathrm{n}$, with some fluctuations and odd-even alternations superimposed on to it. The highest IPs measured were $5.6 \mathrm{eV}$ for $n=6,12$, and 13. This is $0.8 \mathrm{eV}$ below the energy of the ArF excimer laser wavelength. No data exists on the IP of either the dimer or trimer.

To confirm saturation of the photoionization process, power studies of the indium atom and clusters were conducted. Plots of this data are shown in fig. 5 for the atom and some selected clusters. All the cluster saturation curves appeared quite similar to those depicted in fig. 5. The cluster signals appear nearly flat or only slightly increasing with laser power, indicative of saturation. The atom signal also appears saturated at all laser intensity. Since the atom signal is well saturated, the ratio of the atom to cluster signals are lower limits to the actual cluster yield since fragmentation processes can only serve to increase this ratio. It is also noteworthy that the dimer and trimer saturation curves appear similar to all the curves for large clusters. In the absence of more definitive IP data, this fact can be taken as an indication that In 2 and In $_{3}$ must be one photon ionizable by the ArF excimer laser $(6.4 \mathrm{eV})$.

4. Photofragmentation: Since photofragmentation cross-sections and product branching ratios are generally unknown for metal clusters, the measured relative intensities are not necessarily directly proportional to the relative abundance. However, as mentioned above, cluster signal intensities normalizing to saturated atom signals give lower limits to the sputtering yields despite all fragmentation processes. The fact that all clusters were one photon ionized should help to minimize photofragmentation. In addition, modest laser powers $\left(\sim 2 \times 10^{6} \mathrm{~W} / \mathrm{cm}^{2}\right)$ were purposely employed for all measurements in an effort to further 
reduce photofragmentation effects. In assessing if these steps were sufficient, it is encouraging to note that the saturation curves as shown in fig. 5 exhibit no roll over at high laser intensities. Previous measurements, particularly with two photon processes, have reported a roll over effect that has been interpreted to indicate competition between the ionization process and photofragmentation. While special care has been taken to minimize photofragmentation, the saturation data is insufficient prouf to conclude that it is not occurring.

5. Ion Cluster Stability: Ion stability can distort both ion and neutral yield data. This issue is addressed separately from neutral cluster fragmentation because the electronic configuration for neutral and ionic species with the same number of atoms is different. It is possible that secondary ions or photoions will fragment in the free flight path region of SARISA IV and thus will be partially or fully rejected by the band-pass energy analyzer. This is because a daughter ion, even though it has about the same velocity as the parent, will have less kinetic energy due to its smaller mass. This effect is more prominent for small clusters than the large ones since the anergy transmission window for the EARTOF is large and the energy changes due to dissociation are more substantial for small clusters than that for large clusters.

As seen in fig. 4, drops in yield distributions were observed for ionic indium clusters at $n=8,15$ and 16 that are larger than expected from the overall monatomic decrease. This observation is in agreement with the SIMS data of King and Ross.[21] which they interpreted to indicate instability of these ions. What is interesting is that the same structure is observed in our neutral cluster data as can be seen in fig. 3 . This appears to be a clear indication that fragmentation of ionic clusters is occurring prior to detection. An additional point of interest is that the observed drop in signal is about the same for the ionic clusters and neutral clusters despite the fact that there is no reason to anticipate that secondary ions and photoions contain the same amount of internal energy.

None of previously proposed models can successfully explain the variation in power law dependence of the yield on cluster size. For example, the thermodynamic equilibrium model proposed by Urbassek [38] predicts a power law dependence as a function of cluster 
size, but with a constant exponent of $-7 / 3$, and in its present formulation, cannot discriminate between SIMS and SNMS power law dependencies. It is noticed that a recent molecular dynamics and Monte Carlo simulation based on the many-body potential constructed by the embedded-atom method has given a reasonable explanation of the silver sputtering results.[36]

Tractable quantum mechanical models to quantify sputtering from metals, metal oxides and semiconductor surfaces will not be forthcoming in the immediate future; for example, it is still not feasible to employ accurate $a b$ initio calculations at the configuration interaction level to model simple properties of large clusters such as $\operatorname{In}_{32}$ (i.e. geometry, thermodynamic stability and ionization energy). Therefore, there shall ve a continued reliance on molecular dynamics calculation and phenomenological models, the more traditional of which have been successful in simulating one or two aspects of the experimental observations. However, such models must now accommodate an array of observations and so need to be extremely flexible. Observations which are still not fully understood center on: (1) whether the ionic and neutral molecular cluster production process can be described by single or multiple processes and whether separate processes need to be invoked for each class or for each individual cluster; (2) the effect of surface structure on the cluster production process; (3) the effect of fragmentation on the SIMS and SNMS yields; (4) experimental kinetic energy distribution curves, together with the yield information for clusters of a single size. That is, a three dimensional distribution is really required (cluster size, kinetic energy, yield). To further clarify some of these observations in the case of sputtering from polycrystalline indium, work is currently underway to detail the kinetic energy distribution curves of neutral indium clusters as well as to test the effect of surface structure on the production process.

\section{Conclusions}

The bombardment of polycrystalline indium by normal incident $\mathrm{Ar}^{+}$ions produces sputtered clusters as large as $\operatorname{In} 32$ and $\operatorname{In}_{18}^{+}$. The ion to neutral yield is estimated to be on the

order of $10^{-4}$. The In 32 neutral clusters are the largest yet observed from a sputtering process. The yields of both the neutral and ionic clusters fit a power law relationship with slopes of -5.6 
and -4.1 , respectively. Presently, there is no theoretical explanation for the observed power law behavior nor for the empirical observation that the slope of the power law is inversely proportional to the sputtering yield.

\section{Acknowledgments}

The authors would like to thank A. Wucher for helpful discussion S.R.C is grateful for support from a National Science Foundation Graduate Fellowship and the Division of Educational Programs at Argonne National Laboratory. E.vN-F wishes to acknowledge support from the Outside Studies Program of the University of Newcastle, Australia. This work is supported by the U.S. Department of Energy, BES-Materials Science, under Contract W-31-109-ENG-38

\section{References}

[1] M.J. Pellin, C.E. Young, W.F. Calaway, J. E. Whitten, D.M. Gruen, J. D. Blum, I. D. Hutcheon, and G. J. Wasserburg, Phil Trans. R. Soc. Lond. A 333 (1990) 133.

[2] W.F. Calaway, S. R. Coon, M.J. Pellin, C.E. Young J. E. Whitten, R. C. Wiens, D.M. Gruen, G. Stingeder, V. Penka, M. Grasserbauer, and D. S. Burnett, Inst Phys. Conf. Ser. No 128: Section 7, 271.

[3] W. Husinsky, P. Wurz, B. Strehl and G. Betz, Nucl. Instrum. Methods B 18 (1987) 452.

[4] W.H. Christie and D.E. Goering, in: Resonance Ioinization Spectroscopy, Eds. G.S. Hurst and C.G. Morgan, Instrum. Phys. Conf. Ser. 84 (1986) 169.

[5] A.R. Krauss and R.B. Wright, J. Nucl. Mater. 89 (1980) 229.

[6] S.R. Coon, W.F. Calaway, J. W. Burnett, M.J. Pellin, D. M. Gruen, D. R. Spiegel, and J. M. White, Surf. Sci. 259 (1991) 275.

[7] N. Winograd, J.P. Baxter and F.M. Kimock, Chem. Phys. Lett. 88 (1982) 581.

[8] N. Thonnard, J. E. Parks, R. D. Willis, L. J. MOore and H. F. Arlinghaus, Surf. and Interface Anal. 14 (1989) 751.

[9] R. A. Brizzolara ans C. B. Cooper, Nucl. Instrum. Methods B 43 (1989) 136.

[10] H. Gnaser and W. O. Hofer, Appl. Phys. A 48 (1989) 261.

[11] S.R. Coon, W.F. Calaway, M.J. Pellin, and J.M. White, Surf. Sci., in press. 
[12] S.R. Coon, W.F. Calaway, M.J. Pellin, G.A. Curlee and J.M. White, Nucl. Instrum. Methods B 82 (1993) 329.

[13] A. Wucher, M. Wahl, and H. Oechsner, Nucl. Instrum. Methods, in press.

[14] S. R. Coon, W. F. Calaway, and M. J. Pellin, Nucl. Instrum. Methods, in press.

[15] B. Baguenard, M. Pellarin, C. Bordas, J. Lerme', J. L. Vialle, and M. Broyer, Chem. Phys. Lett. 205 (1993) 13.

[16] S.R. Coon, W.F. Calaway, M.J. Pellin, J. W. Burnett, and J.M. White, Surf. and Interface Anal., in press.

[17] F.M. Kimock, J.P. Baxter and N. Winograd, Surf. Sci. 124 (i983) L41.

[18] F.M. Kimock, J.P. Baxter and N. Winograd, Nucl. Instrum. Methods 218 (1983) 287.

[19] B.I. Craig, J.P. Baxter, J. Singh, G.A. Schick, P.H. Kobrin, B.J. Garrison and N. Winograd, Phys. Rev. Lett. 57 (1986) 1351.

[20] M.P. Irion, A. Selinger and R. Wendel, Int. J. Mass Spectrom. Ion Processes 96 (1990) 27.

[21] F. L. King and M. M. Ross, Chem. Phys. Lett. 164 (1989) 131.

[22] C. Kittle, Introduction to Solid-State Physics, 6th Edition (Wiley, New York, 1986).

[23] F. W. Froben, W. Schulze, and U. Kloss, Chem. Phys. Lett. 99 (1983) 500.

[24] R.A. Haring, H.E. Roosendaal and P.C. Zalm, Nucl. Instrum. Methods B 28 (1987) 205.

[25] G.P. Konnen, A. Tip and A.E. De Vries, Rad. Effects 26 (1975) 23.

[26] P. Joyes, J. Phys. B 4 (1971) L15.

[27] J.W. Christiansen, I.S. Tsong and S.H. Lin, J. Chem. Phys. 86 (1987) 4701.

[28] W. Husinsky, G,. Nicolussi and G. Betz, Nucl. Instrum. Methods B 82 (1993) 323.

[29] A. Wucher, M. Wahl, and H. Oechsner, Nucl. Instrum. Methods B 82 (1993) 337.

[30] M. L. Yu, in Sputtering by Particle Bombardment III, eds., R. Behrisch and K. Wittmaack, Vol. 64 of Topics in Applied Physics (Springer-Verlag, Berlin, 1991) p. 91.

[31] Z. Ma, S.R. Coon, W.F. Calaway, M.J. Pellin, D.M. Gruen and E.I. von NagyFelsobuki, to be published.

[32] M. J. Pellin, W. Husinsky, W. F. Calaway, J. W. Burnett, E. L. Schweitzer, C. E. Young, B. Jorgersen, and D. M. Gruen, J. Vac. Sci. Technol. B 5 (1987) 1477.

[33] H. H. Andersen and H. L. Bay, in : Sputtering by Particle Bombardment I, ed. R. Behrisch, Vol. 47 of Topics in Applied Physics (Springer-Verlag, Berlin, 1981) p. 145.

[34] H. Gnaser, M. Saidoh, J. Von Seggern, and W.O. Hofer, Nucl. Instrum. Methods B 15 (1986) 169. 
[35] A. Wucher and B. J. Garrison, Phys. Rev. B 46 (1992) 4855.

[36] A. Wucher, Nucl. Instrum. Methods, in press.

[37] R. C. Weast and S. M. Selby, Handbook of Chemistry and Physics, 48th Edition (The Chemical Rubber Company, Cleveland, 1967) p. e69.

[38] H. M. Urbassek, Nucl. Instrum. Methods B 31 (1988) 541. 


\section{Figure Captions}

Fig. 1 Composite secondary neutral mass spectrum of the sputtered flux from polycrystalline indium.

Fig. 2 Composite secondary ion mass spectrum of the sputtered flux from polycrystalline indium.

Fig. 3 Yield of neutral indium clusters as a function of cluster size.

Fig. 4 Yield of ionic indium clusters as a function of cluster size.

Fig. 5 Secondary neutral signal intensity as a function of laser power for In atoms and clusters containing 5,12 , and 20 atoms. 


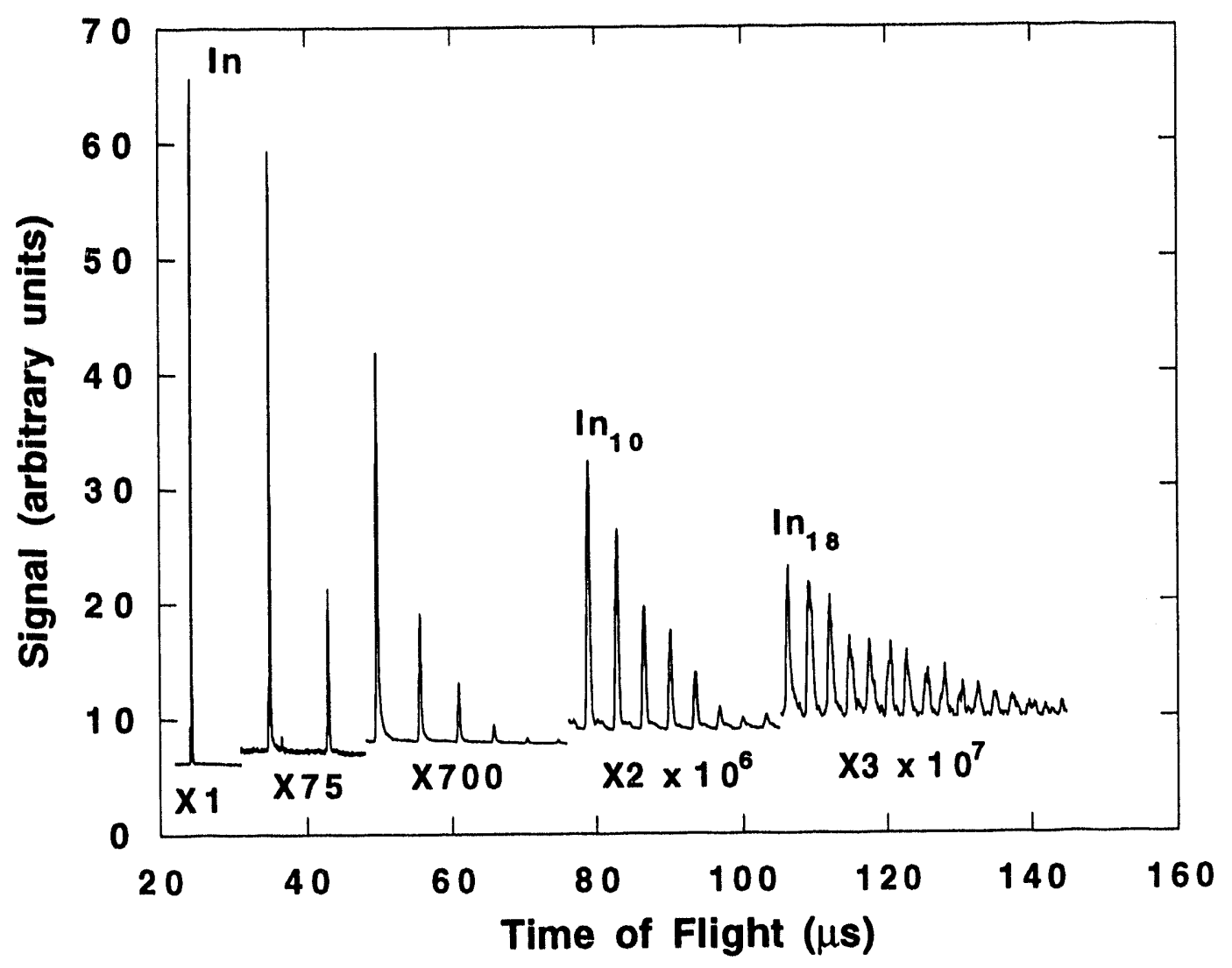




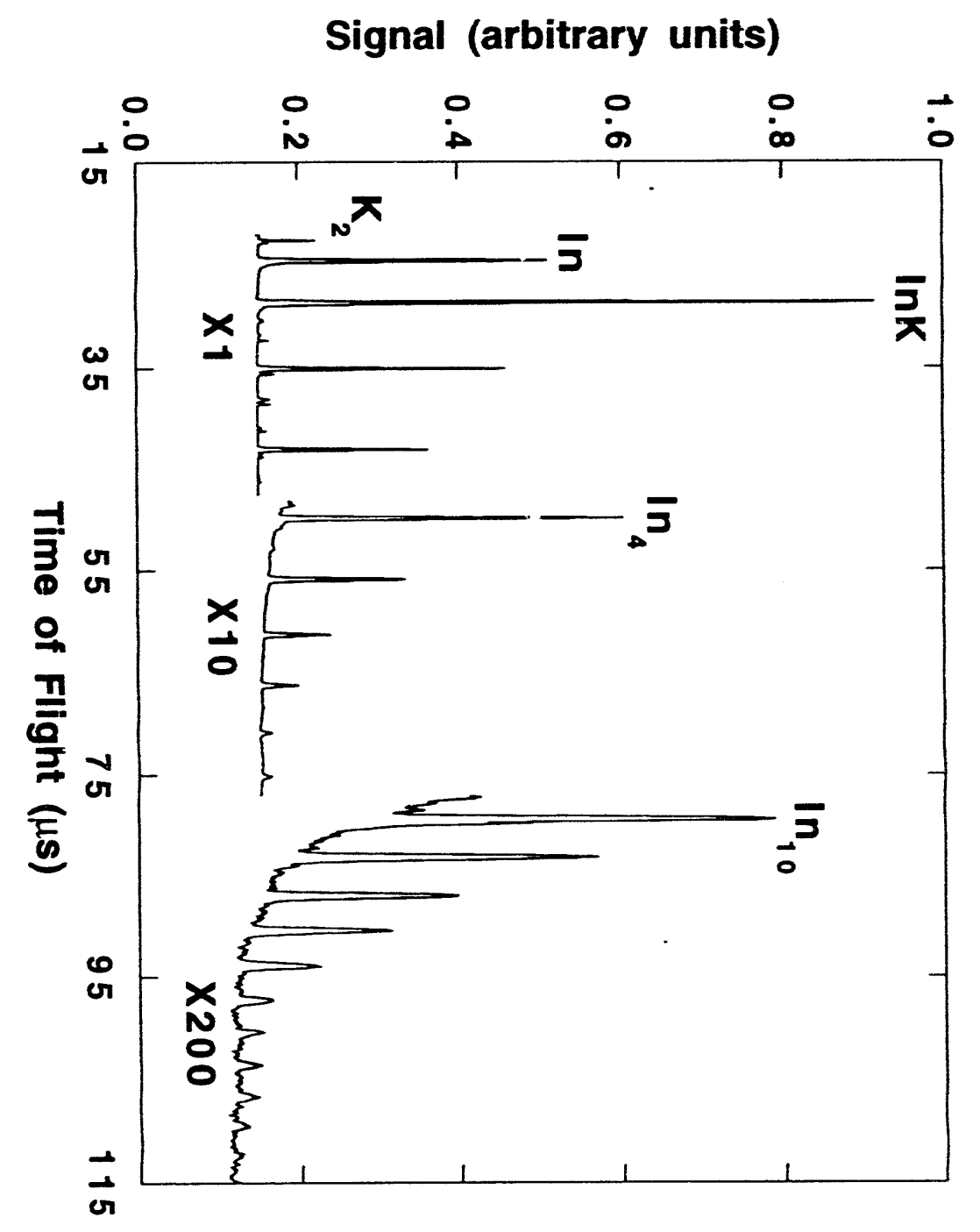




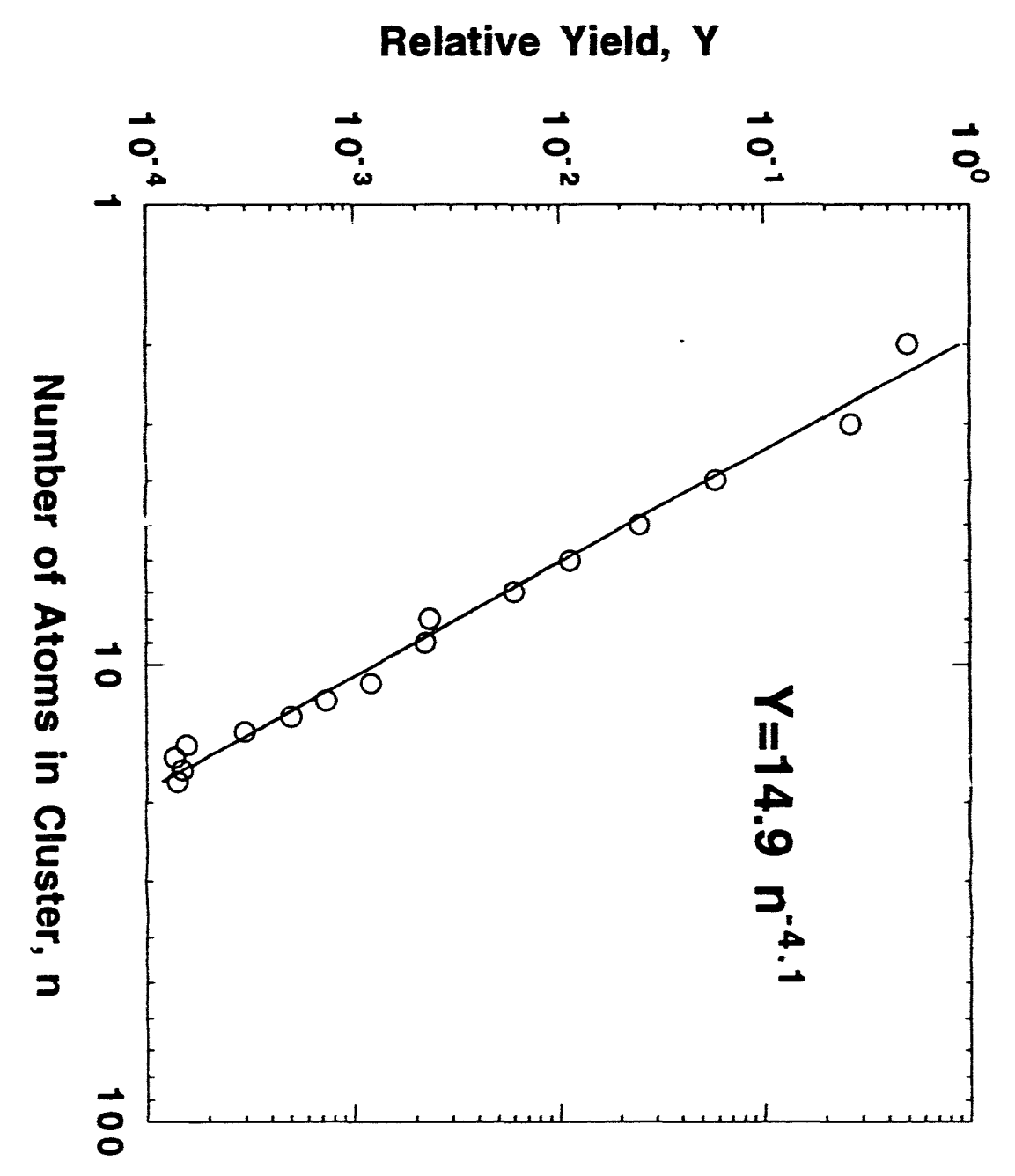




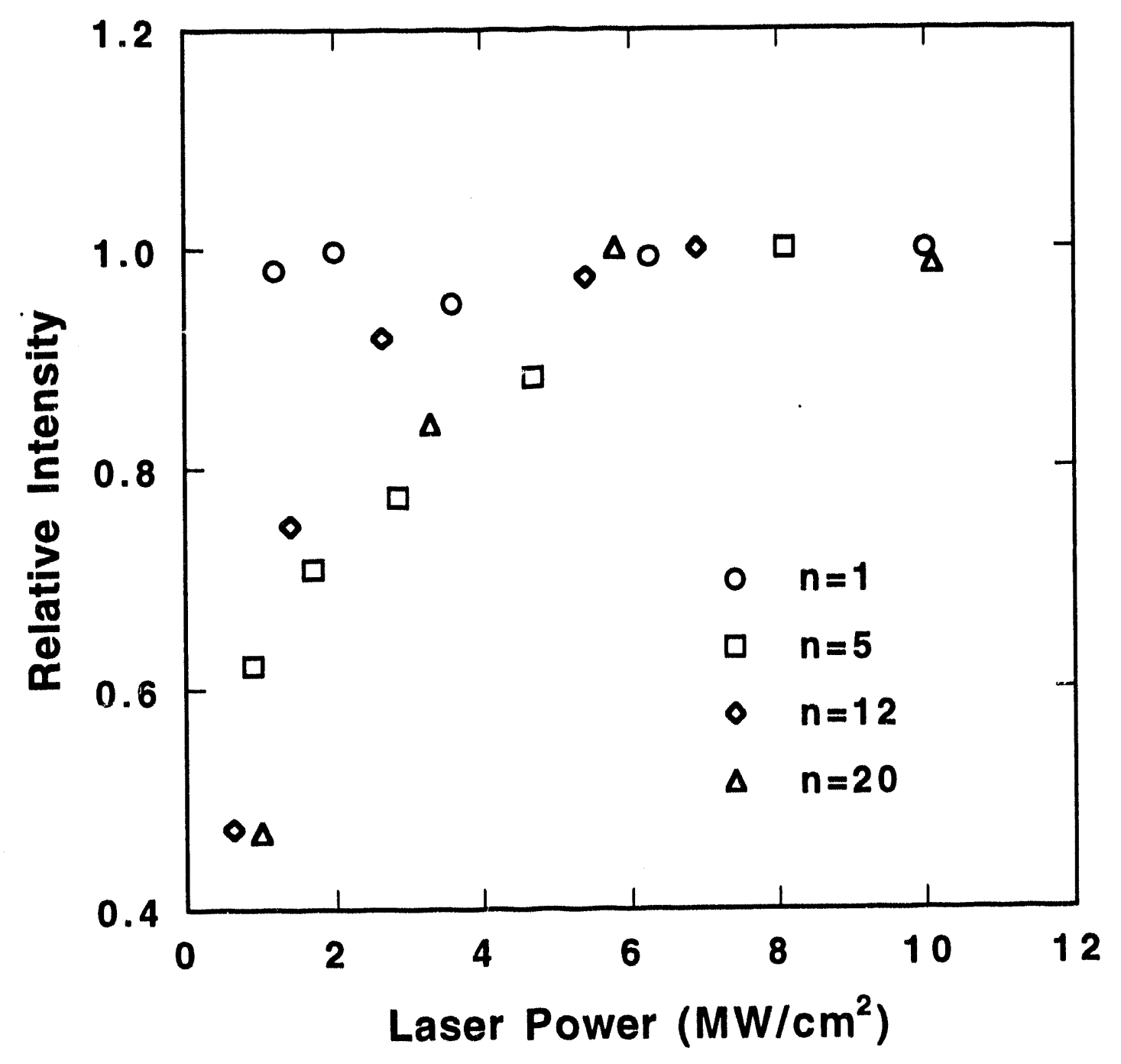



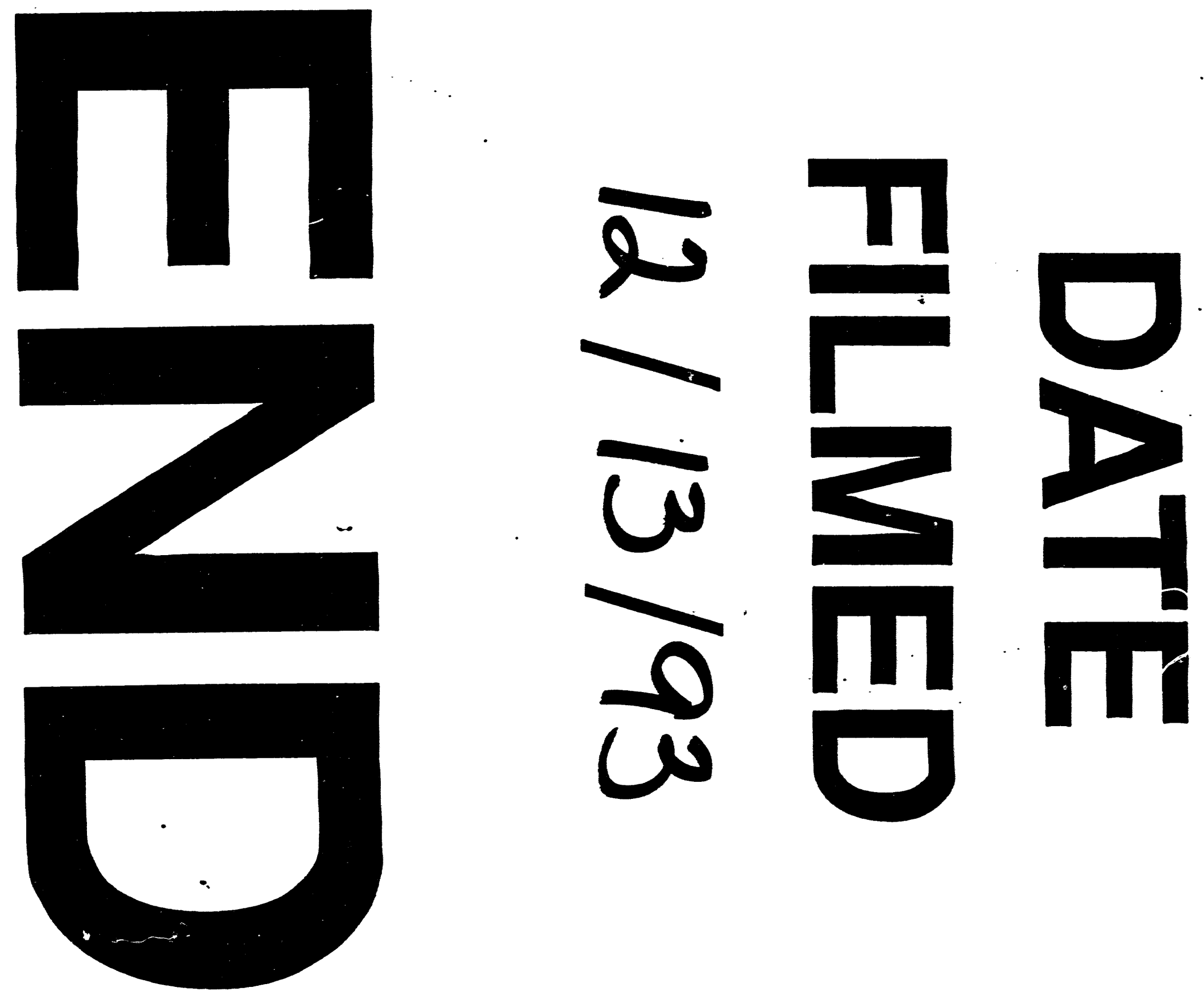
